\title{
Communication
}

\section{Note sur des essais d'immunisation de lapins contre des tsé-tsé, Glossina fuscipes fuscipes (Diptera : Glossinidae)}

\author{
M. Desquesnes ${ }^{1}$
}

DESQUESNES (M.). Note dur des essais d'immunisation de lapins contre des tsé-tsé, Glossina fuscipes fuscipes (Diptera : Glossinidae). Revue Élev. Méd. vét. Pays trop., 1990, 43 (4) : $511-513$

L'immunisation de lapins contre des glossines a été tentée par injection d'intestins ou de jabots homogénéisés de mouches (Glossina fuscipes fuscipes), avec l'adjuvant complet ou incomplet de Freund. L'effet des inoculations est évalué par le suivi de la mortalité et des capacités reproductrices de mouches alimentées sur ces lapins. Les résultats ne montrent qu'une augmentation, peu importante mais statistiquement significative, de la mortalité hebdomadaire dans les lots de glossines nourries sur les lapins immunisés par rapport aux témoins. Mots clés : Lapin - Glossina fuscipes fuscipes - Test d'immunisation.

\section{Introduction}

Certains auteurs ont rapporté soit une mortalité augmentée, soit une fécondité diminuée chez des insectes hématophages nourris sur des animaux d'expérience immunisés avec des extraits de tissus divers d'insecte. ALGER et CABRERA (1) semblent avoir été les premiers à observer une mortalité plus élevée chez des anophèles nourris sur des lapins immunisés avec de l'intestin d'anophèles homogénéisé. SCHLEIN et al. (12) ont pu démontrer que la paroi intestinale d'un insecte (Sarcophaga sp.) est perméable aux immunoglobulines, et attribuent à l'effet des anticorps l'augmentation de la mortalité et les anomalies observées chez des Stomoxys nourries sur des lapins immunisés avec des tissus divers de stomoxes (11). Des glossines, nourries sur ces mêmes lapins, ont subi une plus forte mortalité également ; ils concluent que les anticorps ne sont pas spécifiques. KAAYA et ALEMU (4) ont noté une baisse de la fécondité et une augmentation de la mortalité de leurs pupes, chez des glossines nourries sur des lapins immunisés avec des tissus divers de glossines. Les mêmes auteurs (5) ont également pu constater une augmentation des cas d'anomalies. OTIENO et al. (10) ont rapporté un retardement de la digestion du repas sanguin, mais pas d'augmentation de la mortalité, chez des glossines nourrie sur des lapins immunisés avec un extrait de l'intestin de glossines. Finalement, MATHA et WEISER (9) trouvent que des lapins sur lesquels les glossines sont nourries développent des anticorps contre des antigènes com-

1. Institut d'Élevage et de Médecine Vétérinaire des Pays Tropicaux
(IEMVT), Service d'Entomologie, 10 rue Pierre Curie, 94704 (IEMVT), Service d'Entomolos
Maisons-Alfort Cedex, France.

Adresse actuelle : Institut Pasteur, 97300 Cayenne, France.

Reçu le 27.3.1990, accepté le 11.9.1990. muns aux glandes salivaires et des organes internes divers (y compris l'intestin). MATHA et al. (8) montrent que l'on peut transférer une immunité passive, ayant pour effet une augmentation de la mortalité des glossines avec les sérums de lapins sur lesquels des glossines sont nourries.

L'expérimentation rapportée ci-dessous vise également à reproduire contre la mouche tsé-tsé un mode d'immunisation similaire à celui réalisé contre les tiques, notamment contre la tique du bétail, Boophilus microplus, chez le bovin $(2,6,7)$.

\section{Matériel et méthode}

Les mouches. L'élevage de Glossina fuscipes fuscipes de I'IEMVT (Maisons-Alfort) est réalisé en insectarium climatisé à $15^{\circ} \mathrm{C}$ et $75 \mathrm{p} .100$ d'humidité relative. Les mouches sont alimentées sur les oreilles de lapins, tous les 2 à 3 jours, pendant 5 minutes. Placées dès l'éclosion par lots de 40 femelles et 15 mâles, elles sont ensuite élevées pendant 3 à 4 mois, puis éliminées. Les larves produites sont récupérées tous les 2 à 3 jours, comptées, pesées, et mises en élevage ; les éclosions, environ un mois plus tard, sont notées. La mortalité est observée tous les 3 à 4 jours et les mouches mortes sont éliminées des cages. L'élevage a été décrit en détail par ITARD et BAUER (3).

Les lapins. Neuf lapins de races croisées, d'environ six mois d'âge, d'un poids moyen de $2,5 \mathrm{~kg}$ au début des expériences, ont été utilisés. Ils sont répartis en trois lots (lots A, B et C) de 3 animaux chacun. Pendant 6 semaines, l'élevage des mouches est réalisé sur tous ces lapins neufs afin de les habituer à la manipulation et d'obtenir un niveau stable d'immunisation " naturelle » par les antigènes salivaires, avant de réaliser les immunisations artificielles.

Les vaccins. Les mouches ténérales à jeun sont disséquées dans du PBS. Le jabot et son canal, d'une part, et l'intestin moyen, d'autre part, sont isolés par dissection et selon le cas préparés par dilacération à la seringue, ébullition avec le tampon de Laemli ou sonication pendant 2 minutes suivie par la congélation à - $18^{\circ} \mathrm{C}$. Les protéines sont dosées par la méthode de Bradford. Un volume de $0,5 \mathrm{ml}$ de PBS contenant 20 intestins ( $420 \mu \mathrm{g}$ de protéines) ou 40 jabots $(100 \mu \mathrm{g}$ de protéines) est mélangé à $0,5 \mathrm{ml}$ d'adjuvant complet de Freund (ACF) ou d'adjuvant incomplet de Freund (AIF) ; $0,5 \mathrm{ml}$ du mélange est inoculé en intramusculaire dans les lombes et $0,5 \mathrm{ml}$ en sous-cutanée entre les omoplates.

Le lot $A$ reçoit les préparations à base d'intestins, le lot $B$ est inoculé avec du matériel préparé à partir des jabots, et le lot $C$ reçoit uniquement de l'adjuvant mélangé au PBS. 
TABLEAU I Protocole d'expérimentation.

\begin{tabular}{|c|l|c|}
\hline Injection & Préparation & Adjuvant \\
\hline 1 & Dilacération & ACF \\
2 & Dilacération & AIF \\
3 & Ébullition & ACF \\
4 & Ébullition & AIF \\
5 & Sonication & ACF \\
6 & Sonication & AIF \\
7 & Sonication & ACF \\
8 & Sonication & AIF \\
\hline
\end{tabular}

Chaque lapin reçoit 8 injections, à 3-4 semaines d'intervalle, comme indiqué dans le tableau I: d'abord 2 injections avec les organes dilacérés, ensuite 2 injections avec le matériel porté à ébullition, enfin 4 injections avec les organes soniqués.

\section{Résultats}

Aucun effet direct n'a pu être observé sur les mouches. La production de pupes par rapport au nombre de femelles vivantes n'est pas significativement différente d'un lot à l'autre. Aucune différence n'a pu être décelée entre les lots concernant le taux d'éclosion à 40 jours. De même, la durée d'incubation est identique dans les trois lots.

Ce n'est qu'à partir de la $11^{\circ}$ semaine de l'expérience et durant la $3^{\mathrm{e}}$ semaine suivant la troisième injection vaccinale que les deux lots de mouches, nourries sur lapins vaccinés, ont montré une mortalité hebdomadaire significativement supérieure (au risque de 1 p. 100) à celle du lot témoin. Cette augmentation significative s'est irrégulièrement maintenue au cours des 7 semaines de suivi restant (tabl. II).

\section{Discussion et conclusion}

Certains auteurs ont constaté, lors d'expériencés similaires, une baisse de fécondité sans augmentation de mortalité. D'autres ont obtenu des résultats inverses, comme dans cette étude, où l'immunisation a eu pour conséquence une augmentation de la mortalité, sans baisse de fécondité. L'immunisation des lapins avec du matériel à base d'intestin ou de jabot n'a donné qu'une faible immunité et les chiffres du tableau II ne permettent pas d'envisager avec beaucoup d'espoir une lutte vaccinale contre la glossine (mortalité hebdomadaire au plus 2,6 fois celle du lot des témoins pendant la $16 \mathrm{e}$ semaine par immunisation avec l'intestin, et 2,3 fois celle du lot témoin pendant la 12 e semaine par immunisation avec le jabot).

KEMP et al. (7) ont examiné les perspectives pour la vaccination contre des arthropodes hématophages autres que les tiques. Il existe le plus souvent des barrières entre le sang ingéré et les cellules intestinales : soit la membrane péritrophique, soit un revêtement cuticulaire. La perméabilité de ces barrières aux anticorps peut varier d'une espèce à l'autre, ce qui pourrait diminuer de beaucoup les dégâts causés par la réaction immunologique. D'autres recherches sont nécessaires afin que les contradictions dans les résultats des divers chercheurs soient expliquées et pour que soit déterminé avec plus' de précision quels sont les tissus les plus prometteurs. Des tests d'immunofluorescence pourraient permettre la localisation des anticorps, sur coupes de glossines. Finalement, les études effectuées avec des lapins ne permettent pas d'extrapoler les résultats à d'autres espèces animales.

Remerciements

Nous tenons à remercier MM. CLAIR, MOREL et UILENBERG de l'IEMVT pour leur aide et leurs conseils. Nous sommes également reconnaissant à Mmes DEDEVENS et BERDON ainsi qu'au personnel de l'élevage de glossines de l'IEMVT pour leur collaboration.

TABLEAU II Évolution de la mortalité hebdomadaire des femelles à partir de la septième semaine.

\begin{tabular}{|c|c|c|c|c|c|c|c|c|c|c|c|}
\hline $\begin{array}{l}\text { Semaines } \\
\text { après la } \\
1^{\text {re injection }} \\
\mathrm{N}^{\circ} \text { injection }\end{array}$ & 7 & $\begin{array}{l}8 \\
3\end{array}$ & $\frac{9}{-}$ & $\frac{10}{-}$ & $\frac{11}{-}$ & $\begin{array}{r}12 \\
4\end{array}$ & $\frac{13}{-}$ & $\frac{14}{-}$ & $\begin{array}{r}15 \\
5\end{array}$ & $\frac{16}{-}$ & 17 \\
\hline $\begin{array}{l}\text { Mortalité } \\
\text { hebdomadaire } \\
\text { (p. 100) }\end{array}$ & & & & & & & & & & & , \\
\hline Lot $A$ & 1,4 & 2,8 & 0 & 1,4 & 5,0 & 15,3 & 6,9 & 6,7 & 6,1 & 20,4 & 7,9 \\
\hline Lot $B$ & 0,8 & 3,3 & 1,1 & 1,4 & 3,8 & 17,8 & 8,9 & 9,8 & 6,3 & 13,9 & 5,6 \\
\hline Lot C & 1,4 & 3,3 & 0,6 & 1,4 & 0,9 & 7,8 & 5,9 & 5,2 & 6,0 & 7,8 & 4,7 \\
\hline
\end{tabular}


DESQUESNES (M.). Attempts to immunize of rabbits against tsetse flies Glossina fuscipes fuscipes (Diptera : Glossinidae). Revue Élev. Méd. vét. Pays trop., 1990, 43 (4) : 511-513

Immunisation of rabbits against tsetse flies was attempted by injecting homogenized intestines or crops of these flies (Glossina fuscipes fuscipes) together with Freund's complete or incomplete adjuvant. The effect of the inoculations was assessed by monitoring the mortality and reproductive performance of flies fed on these rabbits. The results show only a modest but statistically significant increase in mortality in the batches of flies fed on immunized rabbits as compared to controls. Key words : Rabbit Glossina fuscipes fuscipes - Immunization test.

\section{Bibliographie}

1. ALGER (N.E.), CABRERA (E.J.). An increase in death rate of Anopheles stephensi fed on rabbits immunized with mosquito antigen. J. econ. Ent., 1972, 65 : 165-168.

2. DESQUESNES (M.), VIGNON (L.). Essai d'un vaccin contre la tique du bétail : Boophilus microplus. (Résultats partiels). Bull. UPRA (Nouvelle-Calédonie), 1987 (12) : 23-28.

3. ITARD (J.), BAUER (B.). Élevages de glossines. Synthèse. Revue Élev. Méd. vét. Pays trop., 1985, 37 ( ${ }^{\circ}$ spécial) : 143-175.

4. KAAYA (G.P.), ALEMU (P.). Fecundity and survival of tsetse maintained on immunized rabbits. Insect Sci. Applic., 1982, 3 : 237-241.

5. KAAYA (G.P.), ALEMU (P.). Further observations on survival and fertility of Glossina morsitans morsitans maintained on immunized rabbits. Insect Sci. Applic., 1984, 5 : 443-446.
6. KEMP (D.H.), AGBEDE (R.I.S.), JOIINSTON (L.A.Y.), GOUGII (J.M.). Immunization of cattle against Boophilus microplus using extracts derived from adult female ticks : feeding and survival of the parasite on vaccinated cattle. Int.J. Parasit., 1986, 16 : 115-120.

7. KEMP (D.H.), PEARSON (R.D.), GOUGH (J.M.), WILLADSEN (P.) Vaccination against Boophilus microplus : localization of antigens on tick gut cells and their interaction with the host immune system. Exp. appl. Acarol., 1989, 7 : 43-58.

8. MATHA (V.), LUKES (S.), SOLDAN (T.). Passive transfer of humoral resistance against adults of the tsetse fly, Glossina palpalis palpalis (Diptera, Glossinidae), in rabbits. Folia Parasit., 1989, 36 : 375-377.

9. MATHA (V.), WEISER (J.). Detection of antigens common to salivary glands and other tissues of tsetse fly, Glossina palpalis palpalis (Diptera : Glossinidae). Folia Parasit., 1988, 35 : 285-287.

10. OTIENO (L.H.), VUNDLA (R.M.W.), MONGI (A.). Observations on Glossina morsitans morsitans maintained on rabbits immunized with crude tsetse midgut proteases. Insect Sci. Applic., 1984, 4 : 297-302.

11. SCHLEIN (Y.), LEWIS (C.T.). Lesions in haematophagous flies after feeding on rabbits immunized with fly tissues. Physiol. Ent., 1976, 1 : 55-59.

12. SCHLEIN (Y.), SPIRA (D.T.), JACOBSON (R.L.). The passage of serum immunoglobulins through the gut of Sarcophaga falculata, Pand. Ann. trop. Med. Parasit., 1976, 70 : 227-230. 